\title{
Fluctuation theorems in driven open quantum systems
}

\author{
Peter Talkner, Michele Campisi, Peter Hänggi \\ Institute of Physics, University of Augsburg, D-86135 Augsburg, Germany \\ E-mail: peter.talkner@physik.uni-augsburg.de
}

\begin{abstract}
.
The characteristic function for the joint measurement of the changes of two commuting observables upon an external forcing of a quantum system is derived. In particular, the statistics of the internal energy, the exchanged heat and the work of a quantum system that weakly couples to its environment is determined in terms of the energy changes of the system and the environment due to the action of a classical, external force on the system. If the system and environment initially are in a canonical equilibrium, the work performed on the system is shown to satisfy the Tasaki-Crooks theorem and the Jarzynski equality.
\end{abstract}

PACS numbers: 05.30.-d,05.70.Ln,05.40-a

Submitted to: Journal of Statistical Mechanics: Theory and Experiment 


\section{Introduction}

About one decade ago Jarzynski proved a quite remarkable equality that relates free energy differences to average exponentiated work done on a thermally insulated system that is acted upon by external time dependent forces varying according to a specified protocol. This equation, now commonly referred to as the Jarzynski Equality, reads [1]:

$$
\left\langle e^{-\beta w}\right\rangle=e^{-\beta \Delta F},
$$

with $w$ the work, $\Delta F$ the free energy difference between a reference equilibrium state of the system at the initial temperature $\beta$ with the force values reached at the end of the force protocol and the truly thermal initial state. Note that the Jarzynski Equality holds irrespectively of whether the system ever reaches this reference equilibrium state.

Crooks [2] later showed that Eq. (1) results from the following work fluctuation theorem:

$$
\frac{p_{t_{f}, t_{0}}(w)}{p_{t_{0}, t_{f}}(-w)}=e^{-\beta(\Delta F-w)}
$$

that relates the probability density function ( $\mathrm{pdf}$ ) of work $p_{t_{f}, t_{0}}(w)$ in the real forward process proceeding from the time $t_{0}$ until $t_{f}$ to the pdf of negative work $p_{t_{0}, t_{f}}(-w)$ of the mirror image process where the time ideally runs backward.

An important question that naturally arises is whether Eqs. (1) and (2) keep holding in the more realistic situation where the system remains in thermal contact with its environment while the forcing protocol is in action. In regard to Eq. (1) a positive answer to this question was already given in Ref. [1] on the basis of classical arguments.

Moreover, quantum extensions of Eqs. (1) and (2) were developed, too, first the quantum version of the Jarzynsky equality for cyclic processes [3], and shortly after the Crooks theorem was demonstrated to hold also for closed quantum systems [4]. A specification of the full statistics in terms of the characteristic function, i.e. the Fourier transform of the probability density function (pdf) of work was obtained in [5], and a proof of the Crooks relation on the basis of this characteristic function was given in Ref. [6]. A generalization to arbitrary initial states such as the microcanonical state was obtained in Ref. [7]. In the latter case a microcanonical Crooks theorem was proved by means of which the change of the thermodynamic entropy can be inferred from the statistics of the work $[7,8]$. Illustrative examples for differently driven quantum harmonic oscillators were discussed in Refs. [9, 10]. An experimental test was proposed in Ref. [11].

Quantum generalizations of the Jarzynski equality and the Crooks theorem for open quantum systems have almost exclusively been studied for systems with Markovian dynamics [12, 13, 14, 15]. Therefore, so far, only systems with weak coupling to their environments have been considered as weak coupling is implicit in the Markovian assumption $[16,17,18,19] \ddagger$.

¥ In the strong coupling limit the dynamics of a particle's position is described by a classical Smoluchowski equation $[20,21,22,23]$ but this equation does not provide information about the 
In the present paper we too restrict ourselves to the case of weak interaction between the system and its environment. We do so because to our best knowledge presently an unambiguous definition of work and heat of a small open quantum system is only known in this very case of weak coupling [24, 25]. However, neither the dynamics of the system nor of the bath is restricted otherwise. In this way we allow for general non-Markovian dynamics imposed by the bath and arbitrary force protocols which are neither constrained to be fast nor slow.

Here we employ the characteristic function approach of Refs. [5, 7] to address the question regarding the validity of the Eqs. (1) and (2) in quantum systems in weak contact with their environment. Hence the focus of this study is on the characteristic function of the joint statistics of simultaneous measurements of system and environmental energies, which are commuting observables. Under the assumption of weak coupling the changes of these two energies imposed by the action of an external force can immediately be related to the changes of internal energy and the heat exchanged with the environment, or, equivalently, to work and heat. Our central result is the derivation of a fluctuation theorem of the Tasaki-Crooks type, for the joint pdfs of either internal energy and heat or, heat and work [see Eq. (32) below]. As a corollary of this theorem, we recover the Jarzynsky equation (1) and the Crooks theorem (2) for the marginal pdf of work. This formally proves that the validity of these relations keeps holding for quantum systems weakly interacting with an environment.

Previous investigations of the Jarzynski equality for open Markovian quantum systems $[13,15]$ are based on the limit of infinitely many repeated measurements of the environment's energy. In contrast, the present approach relies on energy measurements of the system and the environment at the beginning and the end of the protocol. We note that other fluctuation theorems are known in literature for the heat exchange between two systems which either couple directly to each other [26], or through an intermediate system giving rise to a heat flow [27]. In contrast, here we consider systems which initially are in thermal equilibrium with their environment and are then driven out of equilibrium by a classical external force.

The paper is organized as follows. In Sec. 2 we define and obtain the general expression for the joint pdf of internal energy and heat. In Sec. 3 the joint pdf is further evaluated for the initial canonical state of the total system and a fluctuation theorem for work and heat is obtained. Conclusions are drawn in Sec. 4.

\section{Characteristic functions of work, heat and internal energy}

We consider a system $S$ that is in weak contact with its environment $B$. Accordingly, the Hamiltonian $H(t)$ of the total system consists of a system and an environmental part $H^{S}(t)$ and $H^{B}$, respectively, and the interaction Hamiltonian $H^{S B}$, i.e.

$$
H(t)=H^{S}(t)+H^{S B}+H^{B}
$$

dynamics of the momentum. 
where the interaction is small compared to both the system and the environmental Hamiltonians. We assume that the gauge is fixed in such a way that the Hamiltonian $H^{S}(t)$ coincides with the system energy despite its time dependence [28]. This time dependence is caused by an external change of system parameters according to a prescribed protocol. Since the system and the environmental Hamiltonians commute with each other the energies of the system and of the bath can be measured simultaneously with possible results $e_{i}^{S}(t)$ and $e_{\alpha}^{B}$ which are the eigenvalues of the operators $H^{S}(t)$ and $H^{B}$, respectively. The corresponding projection operators onto the common eigenfunctions of these operators are denoted by $P_{i, \alpha}(t)$. Hence, the eigenvalues and eigenprojection operators are determined by the following equations:

$$
H_{S}(t) P_{i, \alpha}(t)=e_{i}^{S}(t) P_{i, \alpha}(t), \quad H_{B} P_{i, \alpha}(t)=e_{\alpha}^{B} P_{i, \alpha}(t) .
$$

The projection operators onto the common eigenspaces of two commuting hermitian operators are hermitian:

$$
P_{i, \alpha}^{\dagger}(t)=P_{i, \alpha}(t),
$$

idempotent and mutually orthogonal:

$$
P_{i, \alpha}(t) P_{i^{\prime}, \alpha^{\prime}}(t)=\delta_{i, i^{\prime}} \delta_{\alpha, \alpha^{\prime}} P_{i, \alpha}(t),
$$

and complete:

$$
\sum_{i, \alpha} P_{i, \alpha}(t)=\mathbb{1}
$$

where $\mathbb{1}$ denotes the unit operator on the total Hilbert space of system and environment. The first measurement is performed at the time $t_{0}$ at which the protocol starts to act. At this very time the state of the total system is assumed to be given by the density matrix $\rho\left(t_{0}\right)$ which we will specify later. The joint probability to measure the respective system and environmental energies $e_{i}^{S}\left(t_{0}\right)$ and $e_{\alpha}^{B}$, in this state is given by

$$
p_{i, \alpha}=\operatorname{Tr} P_{i, \alpha}\left(t_{0}\right) \rho\left(t_{0}\right),
$$

where $\operatorname{Tr}$ denotes the trace over the total Hilbert space of the system and the environment. After a measurement with the outcome $e_{i}^{S}\left(t_{0}\right), e_{\alpha}^{B}$ the system is found in the initial state projected unto the corresponding subspace. This state is given by

$$
\rho_{i, \alpha}=p_{i, \alpha}^{-1} P_{i, \alpha}\left(t_{0}\right) \rho\left(t_{0}\right) P_{i, \alpha}\left(t_{0}\right) .
$$

The second measurement of the system and the environmental energies is performed at the end of the protocol at time $t_{f}$. By then the density matrix of the total system has undergone a unitary time evolution in the total Hilbert space to a new state reading

$$
\rho_{i, \alpha}\left(t_{f}\right)=U_{t_{f}, t_{0}} \rho_{i, \alpha} U_{t_{f}, t_{0}}^{\dagger}
$$

The result of the second measurement is characterized by the conditional pdf of finding energies $e_{i^{\prime}}^{S}\left(t_{f}\right)$ and $e_{\alpha^{\prime}}^{B}$ given that the result of the first measurement were $e_{i}^{S}\left(t_{f}\right)$ and $e_{\alpha}^{B}$. This pdf is given by

$$
p_{t_{f}, t_{0}}\left(i^{\prime}, \alpha^{\prime} \mid i, \alpha\right)=\operatorname{Tr} P_{i^{\prime}, \alpha^{\prime}}\left(t_{f}\right) \rho_{i, \alpha}\left(t_{f}\right) .
$$


Consequently, the joint pdf $p_{t_{f}, t_{0}}\left(\Delta e^{S}, \Delta e^{B}\right)$ to measure changes of the system and the environmental energies $\Delta e^{S}$ and $\Delta e^{B}$, respectively, becomes

$$
\begin{aligned}
p_{t_{f}, t_{0}}\left(\Delta e^{S}, \Delta e^{B}\right)= & \sum_{i, i^{\prime}, \alpha, \alpha^{\prime}} \delta\left(\Delta e^{S}-\left(e_{i^{\prime}}^{S}\left(t_{f}\right)-e_{i}^{S}\left(t_{0}\right)\right)\right) \\
& \times \delta\left(\Delta e^{B}-\left(e_{\alpha^{\prime}}^{B}-e_{\alpha}^{B}\right)\right) p_{t_{f}, t_{0}}\left(i^{\prime}, \alpha^{\prime} \mid i, \alpha\right) p_{i, \alpha} .
\end{aligned}
$$

At weak coupling between the system and the environment the random quantity $\Delta e^{S}$ determines the change of the internal energy of the system. Then the change of the environmental energy equals the amount of energy which is exchanged as heat $Q=-\Delta e^{B}$ with the system. The small contribution of energy that is possibly released from or stored in the interaction Hamiltonian is negligibly small for a system weakly coupled to its environment. Hence, the joint pdf of internal energy change $E=\Delta e^{S}$ and heat exchange $Q$ becomes

$p_{t_{f}, t_{0}}(E, Q)=\sum_{i, i^{\prime}, \alpha \cdot \alpha^{\prime}} \delta\left(E-\left(e_{i^{\prime}}^{S}\left(t_{f}\right)-e_{i}^{S}\left(t_{0}\right)\right)\right) \delta\left(Q+\left(e_{\alpha^{\prime}}^{B}-e_{\alpha}^{B}\right)\right) p_{t_{f}, t_{0}}\left(i^{\prime}, \alpha^{\prime} \mid i, \alpha\right) p_{i, \alpha}$.

The characteristic function $G_{t_{f}, t_{0}}^{E, Q}(u, v)$ provides an equivalent description of the statistics of these energy changes. It is given by the Fourier transform of this pdf, which, due to the presence of the delta functions, can readily be performed to yield

$$
\begin{aligned}
G_{t_{f}, t_{0}}^{E, Q}(u, v) & =\int d E \int d Q e^{i(u E+v Q)} p_{t_{f}, t_{0}}(E, Q) \\
& =\sum_{i, i^{\prime}, \alpha, \alpha^{\prime}} e^{i\left(u\left(e_{i^{\prime}}^{S}\left(t_{f}\right)-e_{i}^{S}\left(t_{0}\right)\right)\right)} e^{-i\left(v\left(e_{\alpha^{\prime}}^{B}-e_{\alpha}^{B}\right)\right)} p\left(i^{\prime}, \alpha^{\prime} \mid i, \alpha\right) p_{i, \alpha} .
\end{aligned}
$$

This expression can be further simplified by means of the Eqs. (8), (12) and the completeness relation (7) into the form of a correlation function, reading

$$
G_{t_{f}, t_{0}}^{E, Q}(u, v)=\operatorname{Tr} e^{i\left(u H_{H}^{S}\left(t_{f}\right)-v H_{H}^{B}\left(t_{f}\right)\right)} e^{-i\left(u H^{S}\left(t_{0}\right)-v H^{B}\right)} \bar{\rho}\left(t_{0}\right)
$$

where the index $H$ denotes the Heisenberg picture of the corresponding operators, i.e.

$$
H_{H}^{S}\left(t_{f}\right)=U_{t_{f}, t_{0}}^{\dagger} H^{S}\left(t_{f}\right) U_{t_{f}, t_{0}}, \quad H_{H}^{B}\left(t_{f}\right)=U_{t_{f}, t_{0}}^{\dagger} H^{B} U_{t_{f}, t_{0}} .
$$

The density matrix $\bar{\rho}\left(t_{0}\right)$ describes the system immediately after the first measurement. It is given by the projection of the initial density matrix $\rho\left(t_{0}\right)$ onto the diagonal states with respect to the eigenbasis of the measured operators. It hence assumes the form

$$
\bar{\rho}\left(t_{0}\right)=\sum_{i, \alpha} P_{i, \alpha}\left(t_{0}\right) \rho\left(t_{0}\right) P_{i, \alpha}\left(t_{0}\right) .
$$

In analogy to the case of the measurement of the total energy of an isolated system the characteristic function for a joint measurement of two energies is determined by a correlation function of the exponential operator $\left(\exp \left[i\left(u H^{S}\left(t_{f}\right)-v H^{B}\right)\right]\right)_{H}\left(t_{f}\right)$ in the Heisenberg picture at the time of the second measurement $t_{f}$ and a second exponential operator $\exp \left[-i\left(u H^{S}\left(t_{0}\right)-v H^{B}\right)\right]$ taken at the initial time. The average is performed with respect to the initial density matrix projected onto the diagonal elements with respect to the joint eigenbasis of the two operators of the first measurement. 
In passing we note that in an analogous way as for the internal energy and the exchanged heat represented by the Hamiltonians of system and environment, respectively, the characteristic function of the changes $x_{i}$ of any mutually commuting set of $N$ observables $X^{i}(t)$ can be represented as a correlation function

$$
G_{t_{f}, t_{0}}^{\left\{x_{i}\right\}}\left(u_{1}, u_{2}, \ldots u_{N}\right)=\operatorname{Tr} e^{i \sum_{i=1}^{N} u_{i} X_{H}^{i}\left(t_{f}\right)} e^{-i \sum_{i=1}^{N} u_{i} X^{i}\left(t_{0}\right)} \bar{\rho}_{0}
$$

where $u_{i}$ denotes the Fourier variable conjugate to $x_{i}, X_{H}^{i}\left(t_{f}\right)$ the observable $X^{i}(t)$ in the Heisenberg picture and where the density matrix $\bar{\rho}_{0}$ is given by the projection of the initial density matrix $\rho_{0}$ onto the common diagonal basis of the observables $X_{i}\left(t_{0}\right)$, $i=1 \ldots N$ as in Eq. (17).

Once the statistical properties of internal energy changes and the exchange of heat are known, the marginal distributions of these quantities can equivalently be characterized in terms of their respective characteristic functions $G_{t_{f}, t_{0}}^{E}(u)$ and $G_{t_{f}, t_{0}}^{Q}(v)$; i.e.,

$$
\begin{aligned}
& G_{t_{f}, t_{0}}^{E}(u)=G_{t_{f}, t_{0}}^{E, Q}(u, 0) . \\
& G_{t_{f}, t_{0}}^{Q}(v)=G_{t_{f}, t_{0}}^{E, Q}(0, v) .
\end{aligned}
$$

Moreover, the internal energy change and the heat exchange determine the work

$$
w=E-Q
$$

performed on the system according to the First Law. Correspondingly, the joint

characteristic function of heat and work, $G_{t_{f}, t_{0}}^{Q, w}(x, y)$, is related to that of internal energy and heat by

$$
G_{t_{f}, t_{0}}^{Q, w}(x, y)=G_{t_{f}, t_{0}}^{E, Q}(y, x-y) .
$$

Then, the marginal characteristic function of work performed on the system becomes

$$
G_{t_{f}, t_{0}}^{w}(z)=G_{t_{f}, t_{0}}^{E, Q}(z,-z) .
$$

So far we have not yet specified the initial density matrix $\rho\left(t_{0}\right)$. In the next section we consider the particularly relevant case of a canonical state of the total system at a given temperature.

\section{Fluctuation theorem for work and heat}

We now assume that the total system consisting of the considered system and its environment is initially, i.e. at $t=t_{0}$, in a thermodynamical equilibrium at inverse temperature $\beta$ and is consequently described by the Gibbs state

$$
\rho_{\beta}\left(t_{0}\right)=Z^{-1}\left(t_{0}\right) e^{-\beta\left(H^{S}\left(t_{0}\right)+H^{B}+H^{S B}\right)},
$$

where

$$
Z\left(t_{0}\right)=\operatorname{Tr} e^{-\beta\left(H^{S}\left(t_{0}\right)+H^{B}+H^{S B}\right)}
$$


denotes the partition function. In the particular case of weak coupling between the system and its environment a perturbation expansion of the density matrix with respect to the interaction Hamiltonian yields up to first order

$\rho_{\beta}\left(t_{0}\right) \approx \rho^{0}\left(t_{0}\right)-Z_{S}^{-1}\left(t_{0}\right) Z_{B}^{-1} \int_{0}^{\beta} d \beta^{\prime} e^{-\left(\beta-\beta^{\prime}\right)\left(H^{S}\left(t_{0}\right)+H^{B}\right)} \delta H^{S B} e^{-\beta^{\prime}\left(H^{S}\left(t_{0}\right)+H^{B}\right)}$,

where

$$
\begin{aligned}
& Z^{S}\left(t_{0}\right)=\operatorname{Tr}_{S} e^{-\beta H^{S}\left(t_{0}\right)} \\
& Z^{B}=\operatorname{Tr}_{B} e^{-\beta H^{S}} .
\end{aligned}
$$

Here $\operatorname{Tr}_{S}$ and $\operatorname{Tr}_{B}$ denote the traces over the system and the environmental Hilbert spaces, respectively. The operator $\delta H^{S B}=H^{S B}-\left\langle H^{S B}\right\rangle_{0}$ specifies the deviation of the interaction from its expectation value with respect to the factorizing state

$$
\rho^{0}\left(t_{0}\right)=Z_{S}^{-1}\left(t_{0}\right) Z_{B}^{-1} e^{-\beta\left(H^{S}\left(t_{0}\right)+H^{B}\right)} .
$$

In order to determine the density matrix $\bar{\rho}\left(t_{0}\right)$, Eq. (17), $\rho_{\beta}\left(t_{0}\right)$ has to be projected onto the diagonal elements with respect to the joint energy eigenbasis of $H^{S}\left(t_{0}\right)$ and $H^{B}$. This projection leaves the unperturbed part $\rho^{0}\left(t_{0}\right)$ of the initial density matrix (25) unchanged. The first order correction on the right hand side of Eq. (25) vanishes in all cases when the interaction between system and environment contains only offdiagonal terms with respect to the unperturbed energy basis as is the case e.g. for the spin-Bose model [29], or the Caldeira-Leggett model [30] $\S$. In all these cases the corrections to the factorizing density matrix $\rho^{0}\left(t_{0}\right)$ are at least of second order in to the system-environment interaction. Therefore they can safely be neglected in the limit of weak coupling such that the diagonal projection of $\rho_{\beta}\left(t_{0}\right)$ leads to the factorizing state $\rho^{0}(t)$, i.e.

$$
\bar{\rho}_{\beta}\left(t_{0}\right)=\rho^{0}\left(t_{0}\right) \equiv Z_{S}^{-1}\left(t_{0}\right) Z_{B}^{-1} e^{-\beta\left(H^{S}\left(t_{0}\right)+H^{B}\right)} .
$$

We emphasize that this holds under the conditions of weak interaction between the system and its environment.

With this initial state the characteristic function for the statistics of internal energy and heat becomes

$G_{t_{f}, t_{0}}^{E, Q}(u, v)=Z_{S}^{-1}\left(t_{0}\right) Z_{B}^{-1} \operatorname{Tr} e^{i\left(u H_{H}^{S}\left(t_{f}\right)-v H_{H}^{B}\left(t_{f}\right)\right)} e^{-i\left(u H^{S}\left(t_{0}\right)-v H^{B}\right)} e^{-\beta\left(H^{S}\left(t_{0}\right)+H^{B}\right)}$.

Analogous to the characteristic function of work performed on a closed system initially in a canonical state [6], this expression can be continued to an analytic function of the variables $u$ and $v$ on stripes $\mathcal{S}_{u}=\left\{u=u^{\prime}+i u^{\prime \prime} \mid u^{\prime} \in \mathbb{R}, 0 \leq u^{\prime \prime} \leq \beta\right\}$ and $\mathcal{S}_{v}=$ $\left\{v=v^{\prime}+i v^{\prime \prime} \mid v^{\prime} \in \mathbb{R},-\beta \leq v^{\prime \prime} \leq 0\right\}$ of the complex plane. The proof is based on the fact that $\exp i u H^{S}(t)$ and $\exp -i v H_{B}$ are trace class operators which are analytical $\S$ If the interaction contains diagonal terms, these can safely be added to the system Hamiltonian, which then would be re-defined accordingly. 
functions of $u$ and $v$ in the specified strips [31]. Setting $u=\bar{u}+i \beta$ and $v=\bar{v}+i \beta$ one finds

$$
\begin{aligned}
Z^{S}\left(t_{0}\right) G_{t_{f}, t_{0}}^{E, Q}(u, v) & =Z^{S}\left(t_{f}\right) G_{t_{0}, t_{f}}^{E, Q}(-\bar{u},-\bar{v}) \\
& =Z^{S}\left(t_{f}\right) G_{t_{0}, t_{f}}^{E, Q}(-u+i \beta,-v-i \beta),
\end{aligned}
$$

where $G_{t_{0}, t_{f}}^{E, Q}(u, v)$ is the characteristic function of the internal energy change and heat transfer for a fictitious process that runs under the action of the time reversed process backward in time. The proof is analogous to the case of a closed system initially in a canonical state [6]. Applying the inverse Fourier transform with respect to both variable $u$ and $v$ one obtains a Tasaki-Crooks type expression $[2,4]$. It establishes a connection between the joint pdf of internal energy change and exchanged heat for the original process to the corresponding quantity of the time reversed process. This fluctuation theorem reads accordingly

$$
\frac{p_{t_{f}, t_{0}}(E, Q)}{p_{t_{0}, t_{f}}(-E,-Q)}=\frac{Z^{S}\left(t_{f}\right)}{Z^{S}\left(t_{0}\right)} e^{\beta(E-Q)}=e^{-\beta(\Delta F-E+Q)},
$$

where $\Delta F=-\beta^{-1} \ln \left[Z^{S}\left(t_{f}\right) / Z^{S}\left(t_{0}\right)\right]$ denotes the free energy difference between the system in the canonical state with the parameter values at $t_{f}$ and in the initial state at $t_{0}$.

\subsection{Nonequilibrium free-energy/work relations}

Replacing the internal energy by the work performed on the system we obtain

$$
Z^{S}\left(t_{0}\right) G_{t_{f}, t_{0}}^{Q, w}(x, y)=Z^{S}\left(t_{f}\right) G_{t_{0}, t_{f}}^{Q, w}(-x-y,-y+i \beta)
$$

or equivalently

$$
\frac{p_{t_{f}, t_{0}}^{Q, w}(Q, w)}{p_{t_{0}, t_{f}}^{Q, w}(-Q,-w)}=e^{-\beta(\Delta F-w)} .
$$

Here $p_{t_{f}, t_{0}}^{Q, w}(Q, w)=p_{t_{f}, t_{0}}(Q+w, Q)$ denotes the joint pdf of heat and work corresponding to the characteristic function $G_{t_{f}, t_{0}}^{Q, w}(x, y)$ defined in Eq. (21). Integrating over all possible values of the exchanged heat one obtains the Tasaki-Crooks theorem for an open system which couples weakly to its environment. It reads

$$
\frac{p_{t_{f}, t_{0}}^{w}(w)}{p_{t_{0}, t_{f}}^{w}(-w)}=e^{-\beta(\Delta F-w)}
$$

where $p_{t_{f}, t_{0}}^{w}(w)=\int d Q p_{t_{f}, t_{0}}^{Q, w}(Q, w)$ denotes the marginal pdf of work. From the TasakiCrooks theorem Jarzynski's work theorem follows immediately saying that the mean value of the exponentiated work performed on an open system in weak contact with its environment coincides with the exponentiated free energy difference [1], i.e.,

$$
\left\langle e^{-\beta w}\right\rangle=e^{-\beta \Delta F} \text {. }
$$




\subsection{Nonequilibrium relations for conditional and marginal probabilities}

From Eq. (32) one can obtain nonequilibrium relations for the marginal distribution of heat

$$
p_{t_{f}, t_{0}}^{Q}(Q)=\int d w p_{t_{f}, t_{0}}^{Q, w}(Q, w)
$$

for the pdf of work $w$ under the condition that the heat $Q$ is measured, i.e.,:

$$
p_{t_{f}, t_{0}}(w \mid Q)=\frac{p_{t_{f}, t_{0}}^{Q, w_{0}}(Q, w)}{p_{t_{f}, t_{0}}^{Q}(Q)},
$$

and for the pdf of heat $Q$ under the condition that the work $w$ is measured, i.e.:

$$
p_{t_{f}, t_{0}}(Q \mid w)=\frac{p_{t_{f}, t_{0}}^{Q, w}(Q, w)}{p_{t_{f}, t_{0}}^{w}(w)},
$$

A straightforward calculation yields:

$$
\frac{p_{t_{0}, t_{f}}^{Q}(-Q)}{p_{t_{f}, t_{0}}^{Q}(Q)}=e^{\beta \Delta F}\left\langle e^{-\beta w} \mid Q\right\rangle,
$$

where the symbol $\langle\cdot \mid Q\rangle$ denotes average over $p_{t_{f}, t_{0}}(w \mid Q)$.

On the other hand one finds the following relation

$$
\frac{p_{t_{f}, t_{0}}(Q \mid w)}{p_{t_{0}, t_{f}}(-Q \mid-w)}=1
$$

for the conditional pdf of heat $Q$ given the work $w$, in the forward and backward processes.

\subsection{Nonequilibrium equalities for exponentiated internal energy and heat}

By noting that

$$
\left\langle e^{\beta Q}\right\rangle_{Q}=\int d Q p_{t_{f}, t_{0}}^{Q}(Q) e^{\beta Q}=G_{t_{f}, t_{0}}^{Q}(-i \beta)
$$

and

$$
\left\langle e^{-\beta E}\right\rangle_{E}=\int d E p_{t_{f}, t_{0}}^{E}(E) e^{-\beta E}=G_{t_{f}, t_{0}}^{E}(i \beta),
$$

and using Eqs. (19) and (30) one finds two nonequilibrium equalities for heat and internal energy:

$$
\begin{aligned}
\left\langle e^{\beta Q}\right\rangle_{Q} & =\frac{\operatorname{Tr} e^{-\beta H^{S}\left(t_{0}\right)} e^{-\beta H_{H}^{B}\left(t_{f}\right)}}{Z_{S}\left(t_{0}\right) Z_{B}} \\
\left\langle e^{-\beta E}\right\rangle_{E} & =\frac{\operatorname{Tr} e^{-\beta H_{H}^{S}\left(t_{f}\right)} e^{-\beta H^{B}}}{Z_{S}\left(t_{0}\right) Z_{B}} .
\end{aligned}
$$

In clear contrast to the Jarzynski equality, the average of the exponentiated heat as well as that of the exponentiated energy do depend on the details of the protocol, i.e. these relations do not depend only on equilibrium properties of the system. 


\section{Conclusions}

We proved that the Tasaki-Crooks fluctuation theorem and the Jarzyinski equality hold in open quantum systems that are weakly coupled to their environments. This was accomplished by introducing the characteristic function for the probability of joint measurements of system and environmental energies, which are commuting observables. This characteristic functions was further evaluated for canonical initial states. Our approach rests on two basic assumptions: First, the total system made of the system of interest and its environment is governed by Hamiltonian dynamics and second, the coupling between system and its environment is assumed to be weak. The first assumption leads to a unitary time evolution of the total system. The second assumption has two important consequences: It allows one to determine the internal energy from the Hamiltonian of the system and the heat from the Hamiltonian of the environment. The work performed on the system then follows by means of the First Law. The contribution of the interaction Hamiltonian is neglected in the definitions of these three energies. The second consequence of the weak coupling assumption is the factorization of the state of the total system immediately after the first measurements of energies into a product of the Gibbs states of the system and the environment as if they were uncoupled up to second order corrections in the system environment interaction. In order for these corrections to be small for weak but nonzero coupling, the temperature of the initial state must not be too low. In contrast, we formally allowed for the exact time evolution such that fast as well as slow protocols can be described adequately.

By an inverse Fourier transformation the joint statistics of internal energy and heat (defined as the energy ceded to the environment) was shown to obey a Tasaki-Crooks type fluctuation theorem. From this we derived the Tasaki-Crooks fluctuation theorem and the Jarzynski equality, for a quantum system that weakly couples to its environment [6]. Further nonequilibrium relations for the marginal and conditional pdfs of work given heat and heat given work were derived.

In the present paper we restricted ourselves to the case where the external forces directly influence the system Hamiltonian but leave the interaction and the environment Hamiltonians unchanged. Our approach can be generalized to more complex situations where the forces immediately influence the system plus environment. In this general case the Tasaki-Crooks theorem, Eq. (34), continues to hold in a slightly modified form: Also the change of the free energy of the environment has to be taken into account, i.e. $\Delta F$ has to be replaced by $\Delta F+\Delta F_{B}$, where $\Delta F_{B}$ denotes the change in free energy of the environment due to the parameter change of the environmental Hamiltonian. In those special cases where only the system and the interaction Hamiltonians are influenced by the external forcing, the form of the Tasaki-Crooks theorem as given by Eq. (34) stays unchanged. As a consequence the Jarzynski equality remains valid for forces changing the system and its interaction to the environment but leaves the Hamiltonian of the environment unchanged.

For a system that strongly couples to its environment it frequently is possible to 
"dress" the system with excitations of its environment such that the dressed system only weakly interacts with its properly redefined environment. Quasi-particles in solid state physics are typical examples of this type of systems. An external force acting on the original system will also be felt by the dressed system. For the work exerted on the dressed system and the heat exchanged with the properly redefined environment all conditions for the presented theory apply and therefore the Tasaki-Crooks theorem and consequently the Jarzynski equality remain valid for such dressed system. Note, however, that for the original "bare" system, as for other strongly coupled systems, these relations cannot be expected to hold.

\section{Acknowledgments}

Financial support by the German Excellence Initiative via the Nanosystems Initiative Munich (NIM) and the Volkswagen Foundation (project I/80424) is greatfully acknowledged.

\section{References}

[1] Jarzynski C, Nonequilibrium equality for free energy differences, 1997 Phys. Rev. Lett. 782690

[2] Crooks G E, Entropy production fluctuation theorem and the nonequilibrium work relation for free energy differences, 1999 Phys. Rev. E 602721

[3] Kurchan G, A quantum fluctuation theorem, 2000 e-print cond-mat/0007360

[4] Tasaki H, Jarzynski relations for quantum systems and some applications, 2000 preprint condmat/0009244

[5] Talkner P, Lutz E and Hänggi P, Fluctuation theorems: Work is not an observable, 2007 Phys. Rev. E 75050102

[6] Talkner $\mathrm{P}$ and Hänggi $\mathrm{P}$, The Tasaki-Crooks quantum fluctuation theorem, 2007 J. Phys. A: Math. Theor. 40 F569-F571

[7] Talkner P, Hänggi P and Morillo M, Microcanonical quantum fluctuation theorems, 2008 Phys. Rev. E $\mathbf{7 7} 05113$

[8] Campisi M, Complementary expressions for the entropy-from-work theorem, 2008 Phys. Rev. E 78012102

[9] Deffner S and Lutz E, Nonequilibrium work distribution of a quantum harmonic oscillator, 2008 Phys. Rev. E $\mathbf{7 7} 021128$

[10] Talkner P, Burada P S and Hänggi P, Statistics of work performed on a forced quantum oscillator, 2008 Phys. Rev. E $\mathbf{7 8} 011115$

[11] Huber G, Schmidt-Kaler F, Deffner S and Lutz E, Employing trapped cold ions to verify the quantum Jarzynski equality, 2008 Phys. Rev. Lett. 101070403

[12] Mukamel S, Quantum extension of the Jarzynski relation: Analogy with stochastic dephasing, 2003 Phys. Rev. Lett. 90170604

[13] De Roeck W and Maes C, Quantum version of free-energy/irreversible-work relations, 2004 Phys. Rev. E 69026115

[14] Esposito M and Mukamel S, Fluctuation theorems for quantum master equations, 2006 Phys. Rev. E 73046129

[15] Crooks G E, On the Jarzynski relation for dissipative quantum dynamics, 2008 J. Stat. Mech.: Theor. Exp. P10023

[16] Grabert H, Weiss U and Talkner P, Quantum theory of the damped harmonic oscillator, $1984 Z$. Phys. B 55 87-94 
[17] Talkner P, The failure of the quantum regression hypothesis, 1986 Ann. Phys. (NY) 167390

[18] Riseborough P, Hänggi $\mathrm{P}$ and Weiss U, Exact results for a damped quantum mechanical oscillator, 1985 Phys. Rev. A 31 471-478

[19] Hänggi H and Ingold G I, Fundamental aspects of quantum Brownian motion, 2005 Chaos 15 026105

[20] Ankerhold J, Pechukas P and Grabert H, Strong friction limit in quantum mechanics: The quantum Smoluchowski equation, 2001 Phys. Rev. Lett. 87086802

[21] Machura L, Kostur M, Hänggi P, Talkner P and Łuczka J, Consistent description of quantum Brownian motors operating at strong friction, 2004 Phys. Rev. E $\mathbf{7 0} 031107$

[22] Coffey W T, Kalmykov Y P, Titov S V, and Mulligan B P, Semiclassical Kramers and Smoluchowski equations for the Brownian motion of a particle in an external potential, 2007 J. Phys. A: Math. Theor. 40 F91-F98

[23] Coffey W T, Kalmykov Y P. Titov S V and Cleary L, Smoluchowski equation approach for quantum Brownian motion in a tilted periodic potential, 2008 Phys. Rev. E $\mathbf{7 8} 031114$

[24] Allahverdyan A E and Nieuwenhuizen T M, Fluctuations of work from quantum subensembles: The case against quantum work-fluctuation theorems, 2005 Phys. Rev. E 71066102

[25] Hörhammer $\mathrm{C}$ and Büttner $\mathrm{H}$, Information and entropy in quantum Brownian motion: Thermodynamic entropy versus von Neumann entropy 2007, e-print arXiv:0710.1716

[26] Jarzynski C and Wójcik D, Classical and quantum fluctuation theorems for heat exchange, 2004 Phys. Rev. Lett. 92230602

[27] Saito K and Dhar A, Fluctuation theorem in quantum heat conduction, 2007 Phys. Rev. Lett. 99 180601

[28] Kobe D H, Gauge-invariant classical Hamiltonian formulation of the electrodynamics of nonrelativistic particles, 1981 Am. J. Phys. 49 581-588

[29] Leggett A J, Chakravarty S, Dorsey A T, Fisher M P A, Garg A, and Zwerger W. Dynamics of the dissipative two-state system, 1987 Rev. Mod. Phys. 59 1-85

[30] Caldeira A O and Leggett A J, Influence of dissipation on quantum tunneling in macroscopic systems, 1981 Phys. Rev. Lett. 46 211-214

[31] Haag R, Hugenholtz N M and Winnink M, On the equilibrium states in quantum statistical mechanics, 1967 Commun. Math. Phys. 5 215-236 